УДК 616.13-77:577.11:59.084:599.323.45

DOI 10.17802/2306-1278-2018-7-2-25-36

\title{
IN SITU VASCULAR TISSUE REMODELING USING BIODEGRADABLE TUBULAR SCAFFOLDS WITH INCORPORATED GROWTH FACTORS AND CHEMOATTRACTANT MOLECULES
}

\section{L.V. Antonova ${ }^{1 \bowtie}$, V.V. Sevostyanova ${ }^{1}$, A.V. Mironov ${ }^{1}$, E.O. Krivkina ${ }^{1}$, E.A. Velikanova ${ }^{1}$, V.G. Matveeva ${ }^{1}$, T.V. Glushkova ${ }^{1}$, Ya.L. Elgudin ${ }^{2}$, L.S. Barbarash ${ }^{1}$}

${ }^{I}$ Federal State Budgetary Institution "Research Institute for Complex Issues of Cardiovascular Diseases», 6, Sosnoviy blvd, Kemerovo, Russian Federation, 650002; ${ }^{2}$ Harrington Heart and Vascular Institute, University Hospitals Cleveland Medical Center, 11100 Euclid Avenue, Cleveland, Ohio 44106, Unites States of America

\section{Highlights}

- The study demonstrates the benefits of using functionalized small-diameter biodegradable vascular graft for de novo vascular tissue formation.

- The release of growth factors and chemoattractant molecules into the vascular bed after the graft implantation stimulates neotissue formation and levels out inflammation and calcification.

$\begin{array}{ll}\text { Background } & \text { incorporation of the growth factors and chemoattractant molecules into the } \\ \text { biodegradable tubular scaffolds can increase their primary patency upon the } \\ \text { implantation into rat abdominal aorta. However, further studies are required to } \\ \text { investigate tissue remodeling using functionalized vascular grafts with the same } \\ \text { diameter as a replaced native vessel. }\end{array}$

Currently, the search for the bioactive molecules capable of promoting formation of the vascular tissue is still ongoing. We have previously demonstrated that incorporation of the growth factors and chemoattractant molecules into the implantation into rat abdominal aorta. However, further studies are required to diameter as a replaced native vessel.

To investigate the specific aspects of de novo vascular tissue formation and

Aim calcification employing rat abdominal aorta interposition model and vascular grafts with $1.5 \mathrm{~mm}$ diameter with incorporated vascular endothelial growth factor (VEGF), basic fibroblast growth factor (bFGF), and stromal cell-derived factor (SDF)- $1 \alpha$.

Tubular grafts with a diameter of $1.5 \mathrm{~mm}$ were blended of poly(3-hydroxybutyrateco-3-hydroxyvalerate) and poly( $\varepsilon$-caprolactone) (PHBV/PCL). Grafts without growth factors were fabricated using standard electrospinning technique whilst grafts with incorporated growth factors were prepared utilizing emulsion

Methods electrospinning. VEGF was incorporated into the inner third, whereas bFGF and SDF-1 $\alpha$ were incorporated into the outer two-thirds of the graft. Grafts were implanted into the abdominal aortas of Wistar rats for $1,3,6$, and 12 months following scanning electron microscopy along with histological and immunofluorescent examination.

Primary patency of the grafts with VEGF, bFGF, and SDF-1 $\alpha$ reached $93 \%$

Results indicative of structural integrity of the vascular tissue. Neither signs of inflammation nor severe calcification was detected.

As in 2 mm diameter vascular grafts, incorporation of bioactive factors into 1.5

Conclusion $\quad \mathrm{mm}$ diameter grafts increased their long-term primary patency and improved vascular tissue formation in comparison with non-modified grafts.

Keywords

Biodegradable polymers $•$ Vascular grafts • Electrospinning • Growth factors • Chemoattractants $\bullet$ Biological activity

Received: 15.03.18; received in revised form: 12.05.18; accepted: 24.05 .18

\section{Список сокращений}

$\begin{array}{ll}\text { bFGF }- \text { basic fibroblast growth factor } & \text { PHBV }- \text { polyhydroxybutyrate / valerate } \\ \text { BSA }- \text { bovine serum albumin } & \text { SDF-1a }- \text { stromal cell-derived factor-1 } \\ \mathrm{Ca}-\text { calcium } & \text { VEGF }- \text { vascular endothelial growth factor } \\ \text { PBS }- \text { phosphate buffered saline } & \text { VWF }- \text { von Willebrand factor } \\ \text { PCL }- \text { polycaprolactone } & \end{array}$




\section{Background}

High prevalence of coronary artery disease and peripheral artery disease leads to a high demand for small-diameter vascular prostheses suitable for surgical revascularization. Tissue engineering of small diameter blood vessels has become a promising approach for generating alternatives to autologous vascular grafts [1]. Therefore, the development of a functionalized biodegradable vascular prosthesis allowing neovessel formation is of paramount importance [1]. We have previously demonstrated that the incorporation of growth factors and chemoattractant molecules directly into the electrospun tubular polymeric scaffold with $2 \mathrm{~mm}$ diameter may enhance its angiogenesis $[2,3]$. Potential benefits of the layered incorporation of several growth factors and chemoattractant molecules into the scaffold and their influence on the cells have been shown [4]. The incorporation of vascular endothelial growth factor (VEGF) into the inner third, and the basic fibroblast growth factor (bFGF) and chemoattractant molecule SDF-1a into the outer $2 / 3$ of the graft wall may stimulate the formation of the endothelial and smooth muscle layers and enhance $100 \%$ patency noted at 12 months after their implantation into the vascular bed of small laboratory animals. The technology of imparting nanoscale internal surfaces to improve adhesion and further vital activity of mature and progenitor endothelial cells has been tested in the grafts with three biologically active molecules. But the small number of the implanted grafts in the previous experiments $(n=$ 4), the mismatch between the diameters of the rat aorta $(\varnothing 1.2 \mathrm{~mm})$ and the graft itself $(\varnothing 2.0 \mathrm{~mm})$ point specific gaps in evidence and set challenges for vascular tissue remodeling using biodegradable scaffolds with improved morphology of the internal surface that we addressed in the current study.

The aim of the study was to assess specific aspects of vascular neotissue formation and calcification evidenced from the long-term implantation of biodegradable vascular grafts with $1.5 \mathrm{~mm}$ dimeter and incorporated VEGF, bFGF and the chemoattractant molecule SDF-1a.

\section{Methods}

Fabrication of functionalized polymeric tubular scaffolds

Tubular matrices with $1.5 \mathrm{~mm}$ diameter containing a complex of biologically active molecules (GFmix) were fabricated by a two-phase electrospinning (Nanon$01 \mathrm{~A}$, MECC, Japan) at a voltage of $23 \mathrm{kV}$, a solution feeding rate of $0.5 \mathrm{ml} / \mathrm{h}$ and $1.5 \mathrm{~mm}$ rotating drum diameter from the composite suspension prepared from a mixture of $5 \%$ polyhydroxybutyrate / valerate (PHBV, Sigma-Aldrich, USA) and $10 \%$ polycaprolactone (PCL, Sigma-Aldrich, USA) dissolved in chloroform and a solution of biomolecules in phosphate buffered saline (PBS). Recombinant rat growth factors (vascular endothelial growth factor (VEGF, Sigma-Aldrich, USA), basic fibroblast growth factor (bFGF, SigmaAldrich, USA) and the recombinant rat chemoattractant molecule SDF-1 $\alpha$ (Sigma-Aldrich, USA) were used as biologically active molecules for the incorporation into the matrices. Biologically active molecules were dissolved in the polymer solution at a ratio of $20: 1$. The final concentration of biomolecules was $500 \mathrm{ng}$ / $\mathrm{ml}$. The inner $1 / 3$ of the scaffold wall was made from the composite suspension of PHBV/PCL and VEGF at $20: 1$ ratio using a $27 \mathrm{G}$ needle to produce more nano-sized polymer filaments on the inner surface of the grafts. Then, the electrospinning was continued using a $22 \mathrm{G}$ needle through which an emulsion of the PHBV/PCL solution with bFGF and SDF- $1 \alpha$ equally diluted in PBS at a final ratio of 20: 1 was fed. The final concentration for each biomolecule was $500 \mathrm{ng} / \mathrm{ml}$ in the polymer solution.

Unmodified biodegradable PHBV/PCL scaffolds were fabricated with electrospinning from a 5\% polyhydroxybutyrate / valerate mixture (PHBV, SigmaAldrich, USA) and 10\% polycaprolactone (PCL, Sigma-Aldrich, USA) dissolved in chloroform without introducing a liquid phase with biologically active molecules in the following mode: applied voltage of $23 \mathrm{kV}$, solution feeding rate of $0,5 \mathrm{ml} / \mathrm{h}$ and rotating drum diameter of $1,5 \mathrm{~mm}$.

\section{In vivo implantation}

6-month-old male Wistar rats weighing 400-450 g $(n=120)$ were used to implant biodegradable vascular grafts. All procedures were carried out in accordance with the principles of the European Convention for the protection of vertebrate animals used for experimental and other scientific purposes (Strasbourg, 1986). All animals received 3\% isoflurane anesthesia which was then maintained with a concentration of $1.5 \%$ isoflurane. Vascular grafts with a diameter of $1.5 \mathrm{~mm}$ and a length of $10 \mathrm{~mm}$ after sterilization by $\gamma$-irradiation were implanted into the rat abdominal aorta by endto-end anastomosis. All surgical procedures were performed under sterile conditions. After surgery, all animals were returned to free access of food and water. Rats with implanted grafts were withdrawn from the experiment at 1, 3, 6 and 12 months $(\mathrm{n}=15$ at every control point; the total number of the PHBV/PCL/ GFmix grafts implanted to the study group was 60 , the total number of the PHBV/PCL grafts implanted to the control group was 60). Polymeric vascular grafts were isolated from the adjacent aorta and divided into two parts. One part was frozen at $-140{ }^{\circ} \mathrm{C}$ for the subsequent immunofluorescent analysis. The second part was fixed in 10\% buffered formalin (Electron Microscopy Sciences) for 24 hours at $4{ }^{\circ} \mathrm{C}$ for the histological assessment. Three grafts from both group at each control point underwent scanning electron microscopy to assess endothelization of the inner graft 
surface and conduct elemental analysis.

\section{Histological examination}

The implanted samples were fixed in formalin for 24 hours, then washed in running tap water, and dehydrated in a graded series of alcohols for 5 hours, then cleared in xylene for 1 hour and in pure xylene for 1 hour. The samples in a mixture of xylene-paraffin were placed in a thermostat overnight (for 16-18 hours) at $37{ }^{\circ} \mathrm{C}$. Impregnation of the samples with paraffin wax ( 3 portions) was performed at $56{ }^{\circ} \mathrm{C}$ for $30-40$ minutes. Impregnated samples were poured into paraffin HISTOMIX (BioVitrum, Russia). All the samples were glued on the block. The embedded samples were cut into 8 - $\mu$ m-thick using a conventional microtome (model HM 325, Thermo Scientific, USA). The samples were then placed in a thermostat and dried overnight at $37^{\circ} \mathrm{C}$. After complete drying of the samples, they were dewaxed in o-xylene (3 portions) for 1-2 min and rehydrated in $96 \%$ alcohol (3 portions) for 1-2 min.

Hematoxin-eosin staining required the dewaxed sections to be washed in distilled water and placed in a Hariss' hematoxylin solution (BioVitrum, Russia) for $15 \mathrm{~min}$, followed by the washing with water for 3-10 min. Subsequently, the sections were immersed in a solution of eosin (BioVitrum, Russia) for $0.5 \mathrm{~min}$, washed in water for $1 \mathrm{~min}$, dehydrated in a graded series of alcohols for $1 \mathrm{~min}$, and cleaned in xylene for 3 min under control. Sections were mounted onto the microscope glasses using the mounting medium.

For Van-Gieson staining, the dewaxed sections were washed in water and placed in Weigert's hematoxylin (BioVitrum, Russia) for 2 minutes, then washed in water until bleaching was achieved. The sections were stained with picrofuxin (BioVitrum, Russia) for 2-3 minutes, then washed in running tap water, dehydrated in a graded series of alcohols up to 5 minutes, cleaned in xylene for several minutes under control while bleaching. Sections were mounted onto the microscope glasses using the mounting medium.

Elastin staining was performed using commercially available kits («Orsein», BioVitrum, Russia). The dewaxed sections were placed in distilled water, then treated with 5 drops of potassium permanganate solution and 5 drops of activating acid buffer for 4 minutes. After that the sections were rinsed with distilled water and treated with 10 drops of oxalic acid for $1 \mathrm{~min}$. After bleaching, the sections were washed twice in distilled water. 20 drops of the alcohol reagent were added to provide the moist chamber. The sections were placed in the container, after being treated with 10 drops of Shikata's orcein, closed and incubated for 30 minutes. The sections were then rinsed in distilled water. Finally, they were treated with 10 drops of the differentiating solution for $2 \mathrm{~min}$ and rinsed in running tap water for $1 \mathrm{~min}$, dehydrated, cleaned and mounted onto the microscope glasses. After that the samples were studied with light microscopy using the AXIO Imager A1 microscope (Carl Zeiss, Germany).

\section{Calcification assessment}

We assessed the presence of amorphous calcium or its crystalline fractions using the sections cut from the explanted PHBV/PCL and PHBV/PCL/GFmix grafts with the diameter of $1.5 \mathrm{~mm}$ which were stained with alizarin red C (Himservice, Russia) and DAPI (SigmaAldrich, USA). In addition, the sections of the PHBV/ PCL/VEGF, PHBV/PCL/bFGF, and PHBV/PCL/SDF1a grafts with the diameter of $2 \mathrm{~mm}$, which long-term patency has been reported previously in $[3,5]$, and the sections of the non-woven PHBV/PCL/VEGF, PHBV/ $\mathrm{PCL} / \mathrm{bFGF}, \mathrm{PHBV} / \mathrm{PCL} / \mathrm{SDF}-1$ a matrices, the respond to their subcutaneous implantation has been studied in [6], underwent the similar staining procedure for the assessment. They were then immersed in a $2 \%$ aqueous solution of alizarin red $\mathrm{C}$ for 70 seconds, then nuclei of cells were stained with DAPI $10 \mu \mathrm{g} / \mathrm{ml}$ for 3 minutes. The specimens were then washed with distilled water, and dried. Cover slips were used to keep the specimens in place. After that, all specimens underwent light and fluorescence microscopy using the AXIO Imager A1 microscope (Carl Zeiss, Germany) with $\times 50, \times 100$ and $\times 200$ magnifications.

\section{Immunofluorescent staining}

The tissue sections of $8 \mu \mathrm{m}$ thickness from the frozen samples were cut with a cryotome Microm HM 525 Cryostat (Thermo Scientific, USA) and mounted on poly-L-lysine coated glasses. Immunofluorescent staining of the sections was performed using the following primary antibodies: mouse anti-rat CD31 (ab119339, Abcam) and rabbit anti-CD34 (ab185732, Abcam), or anti-rat collagen type I antibodies (ab28028, Abcam) and rabbit anti rat collagen type IV antibodies (ab6586, Abcam), or von Willebrand factor antibody conjugated to FITC (ab8822, Abcam). If unconjugated primary antibodies were used, then a mixture of secondary antibodies was applied: goat anti-mouse Ig antibodies, AlexaFluor568 conjugate (ab175473, Abcam), and donkey anti-rabbit Ig antibodies, AlexaFluor488 conjugate (ab150105, Abcam). Autofluorescence was removed with the Autofluorescence Eliminator Reagent (Millipore, USA) according to the manufacturer's instructions. The sections were stained with DAPI (Sigma-Aldrich, USA) and mounted onto the labeled glasses with the ProLong mountant (Life Technologies, USA). Native rat aorta was used as a positive control for anti-CD31 and anti-vWF staining whereas rat embryo was used as a positive control for anti-CD34 staining, and rat skin sections for collagen I and IV. Negative controls were performed by replacing primary antibodies with BSA-PBS while following the staining protocol. The specimens were then analyzed with LSM 700 Confocal 
Laser Scanning Microscope (Carl Zeiss, Germany).

\section{Scanning electron microscopy}

Endothelialization of the internal surface of the explanted vascular grafts was assessed with scanning electron microscopy. First, the samples were fixed with $1 \%$ glutaraldehyde (Sigma-Aldrich, USA) for 1.5 hours, and then postfixed with $1 \%$ osmium tetroxide (Serva Electrophoresis, Germany) in $0.1 \mathrm{M}$ phosphate buffer for 3 hours. Further, the samples were dehydrated in a graded series of alcohols by 15 min incubation with $30 \%$ to $90 \%$ and twice by 30 min incubation at $100 \%$. The samples from both groups were mounted on special tables, then the ion-sputtered method was employed to form conductive coating (gold-palladium coating) using the Emitech SC 7640 (Quorum Technologies, England). The surface of the samples was assessed with a scanning electron microscope (S-3400N, Hitachi, Japan) under high vacuum mode at an accelerating voltage of $5 \mathrm{kV}$ in the secondary electrons imaging.

\section{Statistical analysis}

Statistical analysis was performed using GraphPad Prism (GraphPad Software). A sampling distribution was assessed by the Kolmogorov-Smirnov test. Two independent groups were compared by MannWhitney U-test. More than three independent groups were compared using Kruskal-Wallis test, and the Dunn's t-test was used for a pairwise comparison. P of $\leq 0.05$ were regarded as statistically significant. Data are presented as the median and the 25th and 75th percentiles of Me $(25 \%, 75 \%)$.

\section{Results}

All the animals with the PHBV/PCL implanted grafts survived up to the withdrawal from the experiment. Histological examination reported that $33.3 \%$ (5 out of 15 ) and $26.7 \%$ (4 out of 15 ) of the PHBV/PCL grafts had thrombi partially occluding the graft lumen. There were no signs of the neointima and endothelial layer formation (Fig. 1). Only $66.7 \%$ (10 out of 15 ) and $73.3 \%$ (11 out of 15 ) of the PHBV/PCL grafts were $100 \%$ patent within 1 and 3 months after the implantation. However, the high cellularity of the PHBV/PCL graft walls reported at 1 month developed into moderate granulomatous wall inflammation in $26.7 \%$ of the implanted grafts (4 of 15 ), and moderate neointimal hypertrophy in one case $(6.7 \%, 1$ out of 15$)$ within the 3-months implantation period (Fig. 1). A very thin interrupted neointimal layer proliferated on the inner surface in the rest of the samples. Single cells with the transitional phenotypes $\mathrm{CD} 31+, \mathrm{CD} 34+$ were found within 1 month after the implantation, whereas a discontinuous layer of endothelial cells with the transitional phenotypes CD31+, CD34+ synthesizing von Willebrand factor was determined within 3 months after the implantation (Fig. 2).

Collagen fibers were detected in the graft walls and in the outer capsule (Fig. 1). Collagen type IV was represented by separate inclusions on the inner surface of the grafts (Fig. 2). There were no elastin fibers found (Fig. 1).

All animals who underwent the PHBV/PCL/GFmix graft implantation survived over the 1- and 3-months implantation period. All the PHBV/PCL/GFmix samples (15 out of 15 ) were $100 \%$ patent according to the findings of the morphological examination (Fig. 3).

The inner surface of the grafts was covered with a thin neointimal layer, which further thickened in the zones with calcium crystals within 3 months after the implantation. The graft neointimal surface was covered with the interrupted endothelial layer containing cells with the transitional phenotypes CD31+, CD34+ at 1 month. It suggests the active involvement of hematopoietic progenitor cells attached to the graft surface from the blood flow, which then differentiated into mature endothelial cells and actively expressed the von Willebrand factor (Fig. 4). 3 months after the implantation, the neointimal layer in the PHBV/ PCL/GFmix grafts was covered with a continuous or interrupted endothelial layer with the phenotypes CD31+, and CD34-, actively secreting von Willebrand factor (Fig. 4). A layer of collagen type IV was formed (Fig. 4).

Type I and IV collagen fibers were defined in the graft walls and the outer capsule as well. None elastin fibers were found (Fig. 3, 4). There were no signs of granulomatous inflammation (Fig. 3).

Two animals from the PHBV/PCL group died within $2-2.5$ weeks before the 6-months control point. The cause of death in both cases was the graft thrombosis, accompanied by lower extremity ischemia. When the rest animals were withdrawn from the experiment, the thrombus was found in one more case. It almost occluded the graft lumen. Thus, the 6-months rate of thrombosis was $20.0 \%$ among the PHBV/PCL grafts ( 3 out of 15 ). $80.0 \%$ of the PHBV/PCL grafts (12 out of 15) were patent. However, anterior wall aneurysms were found in $13.3 \%$ ( 2 out of 15 ) of cases. $46.7 \%$ (7 out of 15) of the samples demonstrated moderate granulomatous wall inflammation, which did not interfere in the formation of the elements of the newly formed vascular tissue, but visually increased the cellularity of the graft wall (Fig. 1). The inner surface of the PHBV/PCL grafts was covered with a thin semilunar neointimal layer or had uniformly thin layer. However, the neointima thickened in the zones of the calcium crystal deposits (Fig. 1).

At 6 months control point, all PHBV/PCL/GFmix grafts (15 out of 15) were patent (Fig. 3). Histological examination reported that the inner lumen of each graft was lined with a thin neointimal layer covered with endothelium. There were no signs of neointimal 

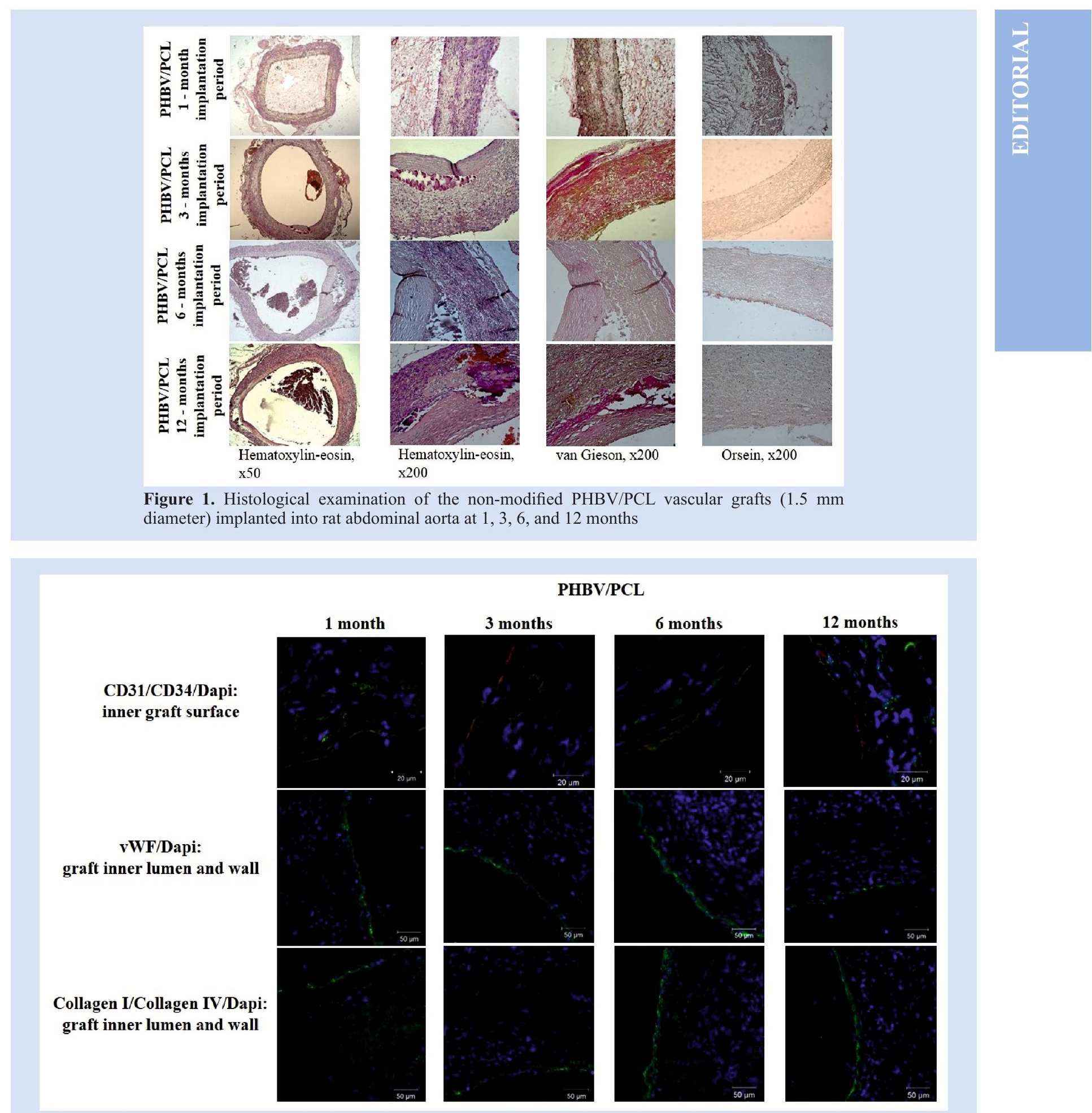

Figure 2. Immunofluorescent examination of the non-modified PHBV/PCL vascular grafts $(1.5 \mathrm{~mm}$ diameter $)$ implanted into the rat abdominal aorta for 1, 3, 6, and 12 months. vWF staining of endothelial cells (green, x200); CD31 staining of mature endothelial cells (red, x630) and CD34 staining of endothelial progenitor cells (green, x630); DAPI staining of nuclei (blue); collagen IV (green, x200) and collagen I (red, x200) staining of extracellular matrix

hyperplasia. Neointima thickened in the zones of the calcium crystal deposits. The cellularity and the development of the connective tissue of the graft wall were moderate and more pronounced in the outer $2 / 3$ of the graft wall (Fig. 3). The inner surface of the modified and non-modified grafts was covered with a layer of mature endothelial cells with the phenotypes CD31+, CD34-, vWF+ (Fig. 2, 4). The layer in the PHBV/PCL grafts was intermittent, whereas in the PHBV/PCL/GFmix grafts it was continuous. A well- defined layer of type IV collagen was detected under the endothelium. In addition, the inclusions of type I and IV collagen were found in the graft wall as well (Fig. 2, 4).

1 animal from the PHBV/PCL group died 6 months before the 12-months control point due to the graft thrombosis, which was accompanied by lower extremity ischemia. 4 more grafts had thrombi according to the histological and immunofluorescent analyses. Thus, $33.3 \%$ of the PHBV/PCL grafts (5 out 

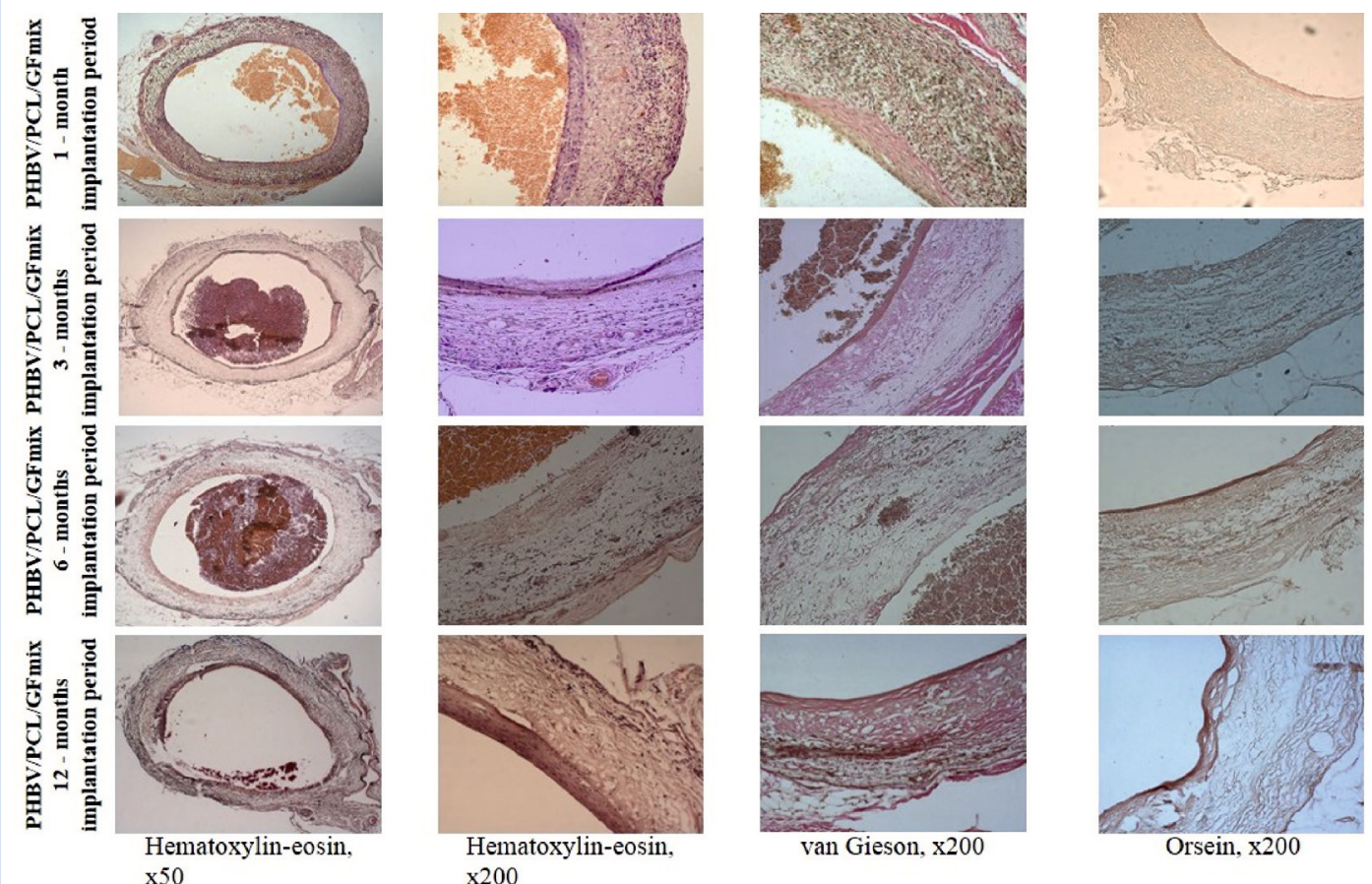

Figure 3. Histological examination of the PHBV/PCL vascular grafts ( $1.5 \mathrm{~mm}$ diameter) with incorporated bioactive factors (VEGF, bFGF, and SDF-1 $\alpha$ ) implanted into the rat abdominal aorta at 1, 3, 6, and 12 months

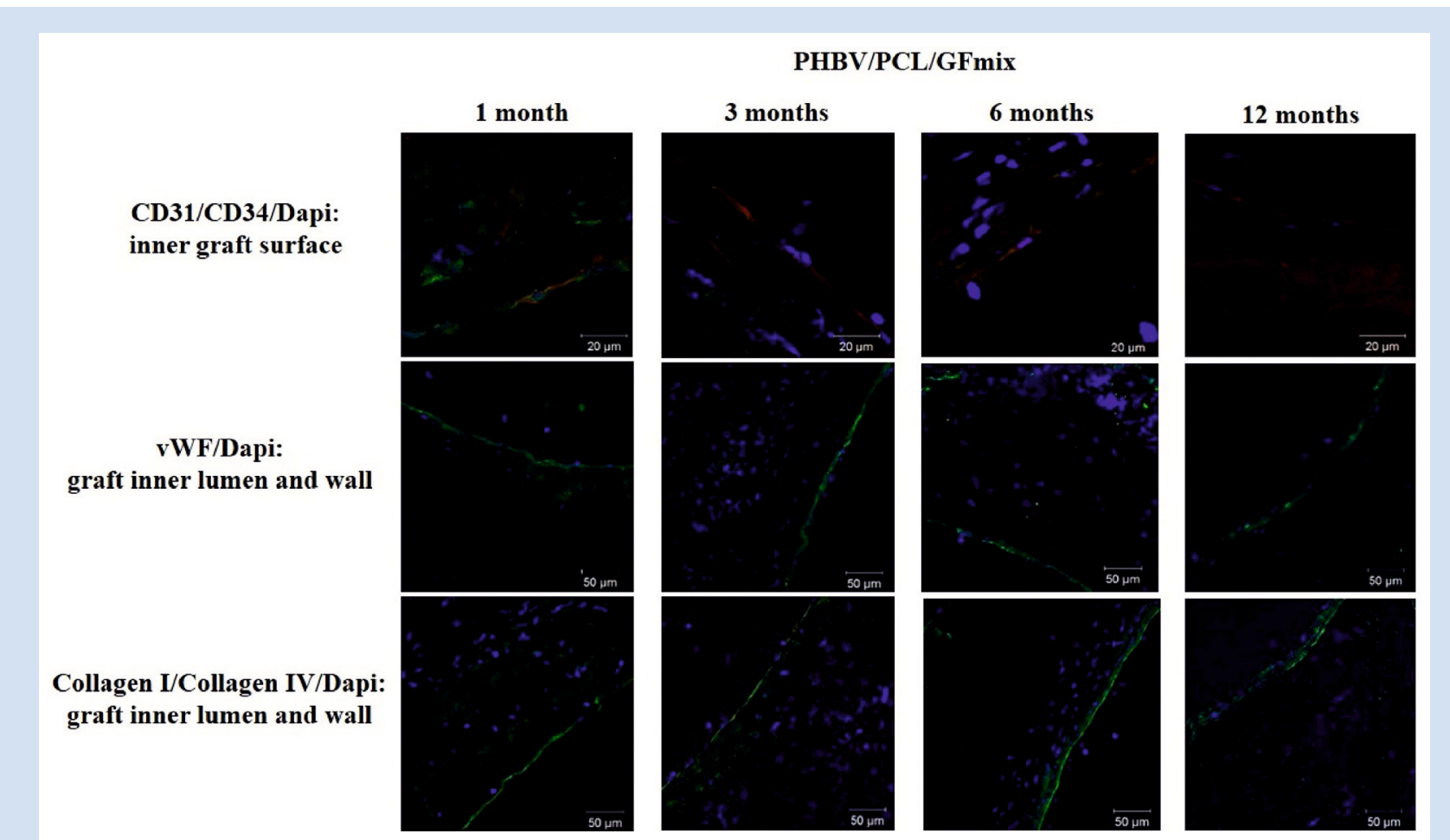

Figure 4. Immunofluorescent examination of the PHBV/PCL vascular grafts (1.5 mm diameter) with incorporated bioactive factors (VEGF, bFGF, and SDF-1 $\alpha$ ) implanted into the rat abdominal aorta at 1, 3, 6, and 12 months. vWF staining of endothelial cells (green, x200); CD31 staining of mature endothelial cells (red, x630) and CD34 staining of endothelial progenitor cells (green, x630); DAPI staining of nuclei (blue); collagen IV (green, x200) and collagen I (red, x200) staining of extracellular matrix

of 15) had thrombi almost occluding the entire graft lumen after the 12-months implantation period. These grafts had reduced wall cellularity, no endothelium and none calcium crystal deposits (Fig. 5). However, $66.7 \%$ of the PHBV/PCL grafts (10 out of 15$)$ were patent (Fig. 1), but $33.3 \%$ of them (5 out of 15 ) had moderate granulomatous wall inflammation, accompanied by a large number of foreign cell bodies, which, however, did not lead to the narrowing of the graft lumen and preventing of the neointima formation, compared to polycaprolactone used for manufacturing a tubular biodegradable scaffold [7]. Due to moderate granulomatous wall inflammation, the cellularity of the non-modified graft walls was visually higher than those in the GFmix grafts. Therefore, the difference in the number of cells in the non-modified and modified 
graft walls suggests the absence of any advantages associated with the non-modified substitutes, but, vice versa, states the problem of an unfavorable cellular response to their implantation. The semilunar neointimal layer covered with endothelial cells was found in all grafts (Fig. 1). Neointimal hypertrophy was observed only in the areas with calcium deposits (Fig. 1). Anterior wall aneurisms were found in 13.3\% of the grafts (2 out of 15) after their explantation.

All animals from the PHBV/PCL/GFmix group survived up to the end of the experiment. Histological and immunohistochemical studies of the explanted specimens reported that $93.33 \%$ of the $\mathrm{PHBV} / \mathrm{PCL} /$ GFmix grafts (14 out of 15) were absolutely patent (Fig. 2). However, 1 graft had an obturating recanalized thrombus occluding the graft lumen. The cellularity of the graft walls was moderate and more pronounced in the outer $2 / 3$ of the walls. There were no cases of granulomatous wall inflammation, compared to the non-modified specimens (Fig. 2).

The inner surface of the PHBV/PCL/GFmix grafts was covered with a thin neointimal layer with a monolayer formed by mature endothelial cells CD31+, CD34-, vWF+ (Fig. 4), whereas an endothelial layer on the inner surface of the PHBV/PCL grafts was intermittent and contained both mature endothelial cells CD31+, CD34-, vWF+ and cells with the transitional phenotypes $\mathrm{CD} 31+, \mathrm{CD} 34+, \mathrm{vWF}+$. It suggested that the last endothelial layer was instable and was constantly renewing (Fig. 4). The subendothelial layer of collagen type IV as well as type I and IV collagen fibers were found in the graft walls and outer capsules of the grafts from both groups (Fig. 2, 4). There were no elastin fibers found at 12 months (Fig. 1, 3).
3 months after the implantation, the deposits of the amorphous and crystalline calcium were first determined in the PHBV/PCL grafts, regardless of the presence or absence of the modification. Thus, $46.7 \%$ of the patent PHBV/PCL grafts (7 out of 15) had amorphous and crystalline calcium foci under the neointimal layer: from single foci to up to $1 / 2$ of the graft circumference (Fig. 5). One small focus was detected only in $6.7 \%$ ( 1 out of 15 ) of the studied specimens, while extended deposits up to $1 / 6$ to $1 / 2$ of the graft circumference were found in $40.0 \%$ of the studied grafts (6 out of 15). The focal deposits of fine-grained crystals of calcium have been determined in $20.0 \%$ of the PHBV/PCL/GFmix grafts (3 out of 15) (Fig. 5). $80.0 \%$ of the grafts (12 out of 15 ) were free of the calcium deposits.

In order to determine the possible pathway of the graft calcification, all grafts with calcium deposits were further divided into 2 groups either with the predominant $\mathrm{Ca}$ deposits in the zone of anastomoses propagating to the central part of the graft wall or uniform Ca deposits along the entire length of the graft, including anastomoses. Only one graft $(6.7 \%, 1$ out of 15) had calcium-enriched anastomosis zone, whereas $40.0 \%$ of the studied grafts (6 out of 15 ) had uniform distribution of $\mathrm{Ca}$ throughout the entire length of the graft. Importantly, 4 patent (26.7\%) and 4 thrombosed $(26.7 \%)$ grafts did not have any calcium deposits.

6 months after the implantation, there were no calcium deposits in the failed grafts $(20.0 \%$ of the total number, 3 out of 15 ) and in $20.0 \%$ of the patent PHBV/ PCL grafts (3 out of 15). 60.0\% (9 out of 15) of the nonmodified grafts demonstrated the deposits of calcium crystals, semilunar or rounded, spread up to $1 / 10-1 / 2$ of

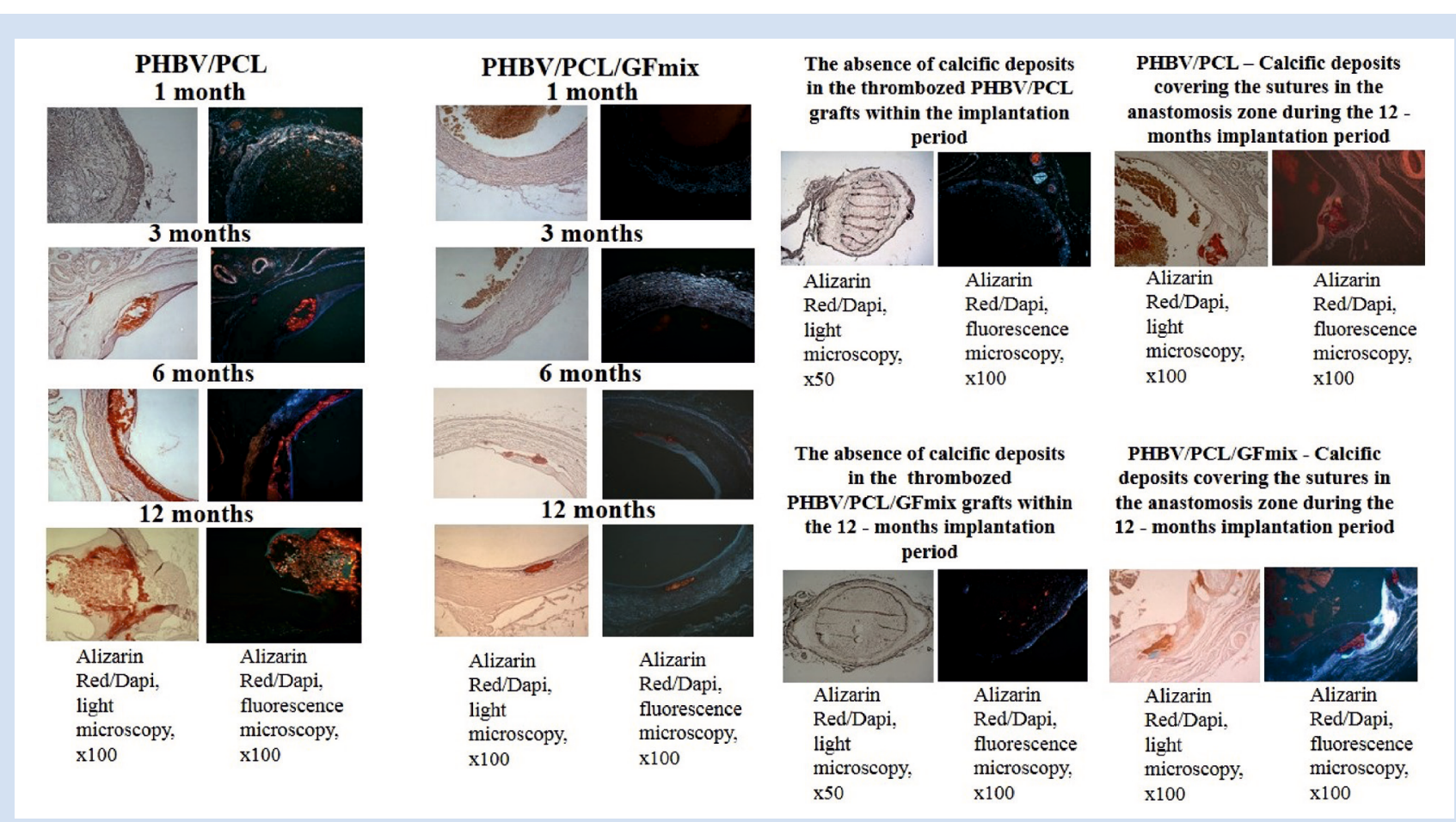

Figure 5. Light and fluorescence microscopy of the non-modified PHBV/PCL vascular grafts and PHBV/PCL vascular grafts with incorporated bioactive factors after combined alizarin red S and DAPI staining for calcium (red) and nuclei (blue), respectively 
the graft circumference (Fig. 5). Nevertheless, calcium deposits in the anastomosis zone and around the suture material prevailed in $13.3 \%$ (2 out of 15$)$ of the studied grafts, whereas calcium deposits throughout the graft length were found in $46.7 \%$ of the PHBV/PCL grafts (7 out of 15).

There were no $\mathrm{Ca}$ deposits in $20.0 \%$ of the explanted PHBV/PCL/GFmix grafts (3 out of 15) within 6 months after the implantation. $80.0 \%$ of the grafts had calcific walls and anastomoses. The more pronounced calcification of the anastomosis zones was found in $13.3 \%$ of the PHBV/PCL/GFmix grafts ( 2 out of 15$)$, including $\mathrm{Ca}$ deposits around the suture material (Fig. 5). 66.7\% of the grafts (10 out of 15) had uniform calcification throughout the entire length and in the zones of anastomoses. Importantly, one small Ca site in the graft wall was detected in $46.7 \%$ of the studied grafts ( 7 out of 15), and two or more foci, or the distribution of crystalline calcium up to 1.8 of the graft circumference was determined in $33.3 \%$ of the PHBV/ PCL/GFmix grafts (5 out of 15). Thus, the grafts with single $\mathrm{Ca}$ foci prevailed in the modified group within 6 months after the implantation.

12 months after the implantation, the failed grafts did not have any signs of calcification among the PHBV/PCL grafts. Large-grained crystals of calcium were detected in all patent grafts (Fig. 5): $13.3 \%$ of the grafts (2 out of 15) had one large focus of crystalline calcium underneath the neointimal layer, and 53.3\% of the grafts (8 out of 15) had semilunar deposits of crystalline calcium from $1 / 8$ to $1 / 2$ of the graft circumference, or multiple individual foci along the entire circumference under the neointimal layer. 26.7\% of the grafts (4 out of 15) had large Ca deposits in the anastomosis zones, whereas $40.0 \%$ of the grafts (6 out of 15) had heavy calcification throughout the entire length of the grafts, including anastomoses.

There were no signs of calcification in $26.7 \%$ of the patent PHBV/PCL/GFmix grafts (4 out of 15) and in $6.67 \%$ of the failed PHBV/PCL/GFmix grafts (1 out of 15) within 12 months after the implantation. Two patent grafts without any signs of the wall calcification had $\mathrm{Ca}$ deposits mainly around the suture material in the anastomosis zones (Fig. 5). 66.7\% patent PHBV/PCL/ GFmix grafts (10 out of 15) had crystalline Ca located underneath the neointimal layer (Fig. 5). The size and extent of the calcium deposits varied significantly from single small foci to the severe calcification throughout the entire graft length. One small focus was found in $26.7 \%$ of the grafts ( 4 out of 15 ), whereas $40.0 \%$ of the $\mathrm{PHBV} / \mathrm{PCL} / \mathrm{GFmix}$ grafts (6 out of 15 ) had several and large deposits.

The layered cutting performed in $26.7 \%$ of the PHBV/PCL/GFmix grafts (4 out of 15) allowed tracing the pattern of calcium distribution from the anastomosis zone, where calcium deposits were mostly accumulated, to the center of the graft, where they were mostly represented by single small foci located under the neointimal layer. However, it was impossible to determine the vector of $\mathrm{Ca}$ spreading into the graft stratum in $40.0 \%$ of the $\mathrm{PHBV} / \mathrm{PCL} / \mathrm{GFmix}$ grafts as the calcium deposits were almost identical in various parts of the grafts, ranging from single foci to insignificant deposits of amorphous $\mathrm{Ca}$ throughout the entire graft length.

Scanning electron microscopy of the inner graft surface was performed to assess endothelization of the explanted vascular grafts with $1.5 \mathrm{~mm}$. 1 month after the implantation, the PHBV/PCL/GFmix grafts had more pronounced endothelization of the inner surface, covering almost the entire studied area. Importantly, there were no exposed areas of the polymer surface, as in the PHBV/PCL grafts. 3 months after the implantation, a continuous endothelial lining covered the entire inner surface of the GFmix grafts was observed. Its integrity preserved until the end of the experiment. The endothelial lining of the nonmodified grafts appeared only within 6 months after the implantation and was equal to that in the PHBV/PCL/ GFmix grafts. However, the degree and uniformity of the endothelial lining in the area of anastomoses were higher in the PHBV/PCL/GFm grafts since they were similar to the inner surface of the adjacent segments of the native aorta (Fig. 6).

The experiment suggested that calcification developed only in the patent grafts. It means that blood, being a biological medium saturated with cells and calcium, and the blood flow, supports and / or provokes the development of calcification processes. This assumption is further supported by the fact that the failed non-modified and modified PHBV/PCL grafts did not have any calcium deposits.

To confirm this assumption that blood as a biological medium and blood flow capable to mediate mechanotransduction trigger the development of prosthesis calcification after its implantation into the vascular bed, additional studies were conducted to detect calcium in the samples of non-woven matrices implanted subcutaneously for a 12-months period (Fig. 7). Therefore, we analyzed the explanted samples of the non-woven PHBV/PCL matrices with incorporated VEGF, bFGF and SDF-1a. The tissue reaction to the subcutaneous implantation of these matrices has been previously discussed in [6]. In addition, we used other vascular graft samples with $2 \mathrm{~mm}$ diameter containing VEGF, bFGF, SDF-1a which were previously implanted for a 12-months period in our previous experiments [2, $3]$ to assess the impact of the modification approach of biodegradable vascular grafts on their calcification. These samples were additionally stained with alizarin red and DAPI and subjected to fluorescence microscopy (Fig. 7).

We found that there were no calcium deposits in the samples of non-woven matrices implanted subcutaneously within the 12-months period (Fig. 7). 
Despite the modification approach, calcium crystals were found in all explanted samples of the PHBV/ PCL vascular grafts of $2 \mathrm{~mm}$ in diameter. The calcium crystals were mainly located under the neointimal layer and around the suture material (Fig. 7).

Thus, the incorporation of the growth factors and chemoattractant molecules into the biodegradable tubular scaffolds, at least in an isolated form, does not provoke calcification processes that starts in the graft wall after their implantation into the vascular bed of small laboratory animals. A key role of the blood flow in the initiation of calcification processes is indirectly evidenced by the results of the 12-month subcutaneous implantation of the same matrices and the absence of any $\mathrm{Ca}$ deposits in the failed grafts.

\section{Discussion}

Rapid endothelization, the formation of smooth muscle cells and a large number of cells that produce an intercellular matrix are the decisive factors that ensure

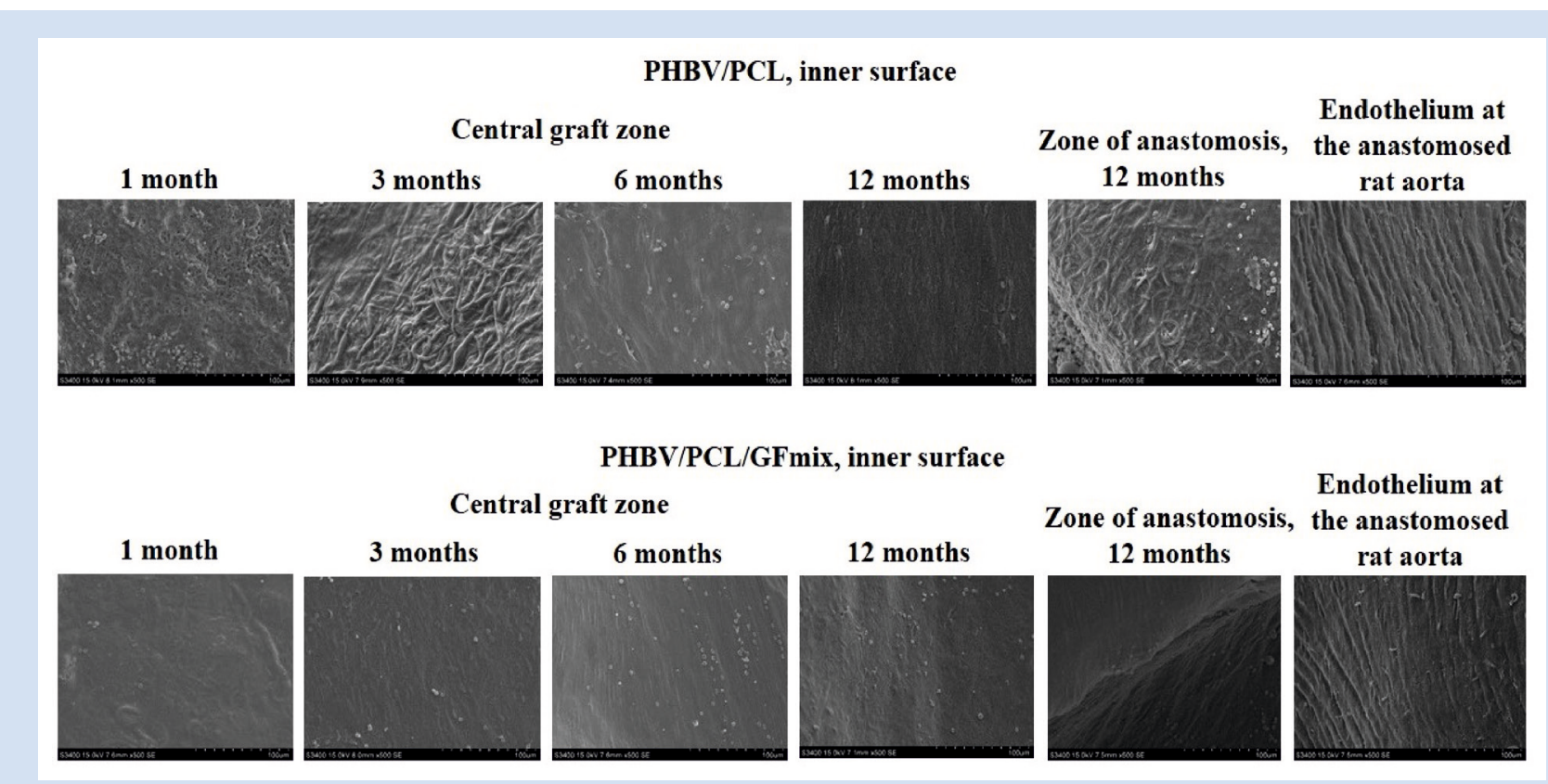

Figure 6. Scanning electron microscopy of the luminal surface of the non-modified PHBV/PCL vascular grafts and PHBV/PCL vascular grafts with incorporated bioactive factors along with an adjacent aortic segment, x500

2-mm biodegradable PHBV/PCL grafts with incorporated growth factors and chemoattractant molecules.

Graft implantation period into rat aorta -12 months.
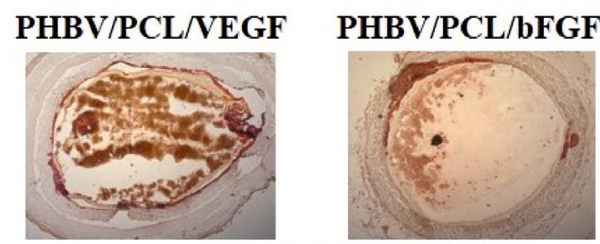

Alizarin Red/Dapi, light microscopy, x50
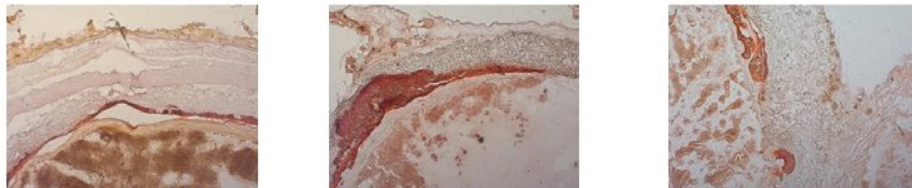

Alizarin Red/Dapi, light microscopy, x100
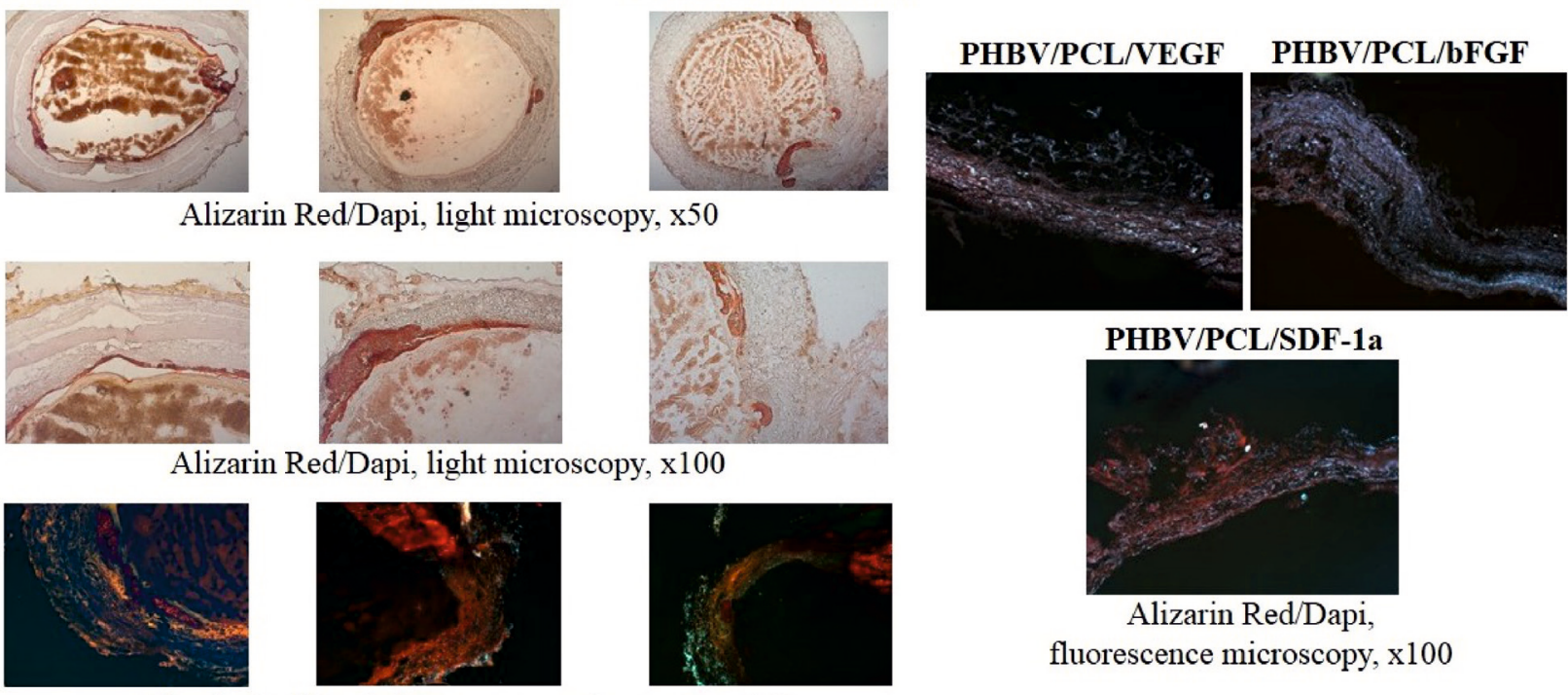

PHBV/PCL/SDF-1a

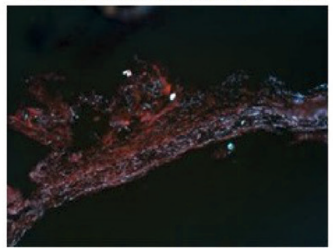

Alizarin Red/Dapi,

fluorescence microscopy, x100

Alizarin Red/Dapi, fluorescence microscopy, x100

Figure 7. Light and fluorescence microscopy of $2 \mathrm{~mm}$ diameter PHBV/PCL vascular grafts with incorporated bioactive factors upon 12-month intraaortic and subcutaneous implantation. Combined alizarin red S and DAPI staining for calcium (red) and nuclei (blue), respectively 
high primary patency of vascular grafts $[8,9,10]$. Importantly, the PHBV/PCL/GFmix grafts were $100 \%$ patent within the 12 -months implantation period. GFmix contributes to the active sticking of precursor cells from the bloodstream to the inner surface of the grafts, which led to the formation of the mature endothelial cell layer within the 3-months implantation period. There was no granulomatous wall inflammation in the PHBV/PCL/GFmix grafts. However, the cellularity of the grafts was inferior to the non-modified substitutes, which may be explained with the increased nanostructure of the inner graft surface and limited cell migration into the thickness of the tubular scaffolds. The similar reason could affect the absence of a visible difference in the formation of the connective tissue in the modified and non-modified grafts represented by type I and IV collagens. The total patency of the PHBV/PCL/GFmix vascular grafts within the 12 -months implantation period was $93.33 \%$ due to the stable endothelization of the inner graft surface in situ. 12 months after the implantation of the non-modified PHBV/PCL vascular grafts, there were signs of endothelial layer desquamation and endothelial population renewal with moderate granulomatous wall inflammation observed in $33.3 \%$ of the grafts, which resulted in the preservation of the primary patency in $66.7 \%$ of the PHBV/PCL grafts.

Pathological calcification of the cardiac structures is one of the main challenges of modern medicine. Despite the fact that the mechanisms underlying these processes are poorly understood [11], researchers agree that the initiating factors of calcification include endothelial dysfunction, inflammatory reactions and oxidative stress, leading to remodeling and mineralization of cardiac structures [12, 13]. Recent studies have shown that cardiovascular calcification is triggered through the activation of cellular processes in the presence of an uncontrolled pathological environment that cannot be reproduced in the in vitro models. Therefore, it limits the opportunities to investigate the cause-effect relationships, mostly studied by the endpoints of the affected tissues [14, 15]. Smooth muscle cells are considered to be the main source of origin of osteogenic cells in calcified vascular lesions [16]. Other data indicate that the occurrence of ectopic medial calcifications is associated with adventitious cells [17]. Vessel and valve endothelial cells serve as a cellular barrier between the blood and the main tissue components, but participate in the endothelial-mesenchymal transition, playing a key role in tissue fibrosis and calcification [18]. In vitro and ex vivo studies have established the involvement of inflammation in the calcification of both valves and vessels $[19,20]$. Calcification in these tissues follows the accumulation of macrophages, and its final stage is accompanied by the induction during inflammation [14]. Importantly, a new concept suggesting that progenitor cells circulating in the blood under certain conditions can exhibit their osteogenic potential and promote intimal [21] and valvular calcification [22] has recently emerged. It was shown that with increasing progression of aortic valve stenosis, the number of circulating osteocalcin-positive endothelial progenitor cells $(\mathrm{CD} 34+/ \mathrm{OCN}+)$ increased with their subsequent accumulation in the calcified aortic valve tissue [22]. Thus, this cascade results in the deposition of calcium, primarily in the zones with critical stress [14].

The hypothesis of mechanical stress, as a factor contributing to the onset of calcification, has been confirmed in several studies indicating that the accumulation of calcium deposits is mainly associated with the areas with increased stress experienced by tissues during functioning under pulsatile flow [23]. Similar patterns have been described both in clinical studies [14] and in in vitro studies [24]. However, some researchers state that dynamic stress promotes the development of calcification, but it is not its primary cause [13]. Suture material may be considered an additional factor contributing to the calcification of prosthetic substitutes [25], which in our experiments was not used for subcutaneous implantation of the nonwoven matrices, but was used for placing anastomoses during the implantation of biodegradable vascular grafts. Thus, calcification of both cardiac structures and implanted devices is more likely to be the endproduct of cellular processes and resultant stresses under pulsatile flow.

The comparative analysis of the nature and prevalence of calcification of the non-modified and modified graft walls, almost a third of the explanted grafts showed that the onset of calcification processes was triggered by anastomoses and surgical suture material. There were no differences in the severity of $\mathrm{Ca}$ deposits in the various zones among $40 \%$ of the grafts. However, more severe calcification prevailed in the non-modified grafts. $53.3 \%$ of the grafts had semilunar crystalline calcium deposits located beneath the neointima and occupied almost half of circumference of the graft wall.

The absence of calcification in both, the non-woven PHBV/PCL matrices during subcutaneous implantation and thrombosed biodegradable vascular grafts, confirms the assumption that blood being an aggressive biological medium and pulsatile flow provoke calcification of the implanted grafts. An additional factor that may also contribute to the onset of the graft wall calcification is the process of biodegradation accompanied by the activation of cells of the monocytemacrophage system, which are actively involved in the processes of developing inflammatory response, for example, granulomatous inflammation. Graft wall biodegradation of the thrombosed and patent PHBV/ PCL vascular grafts is associated with the activity of cells of the monocyte-macrophage system and does not 
reliant on the presence or absence of blood flow. Therefore, the absence of $\mathrm{Ca}$ deposits in the thrombosed grafts indicates that cellular reactions accompanying biodegradation processes are not involved in the onset and maintenance of calcification processes.

\section{Conclusion}

Thus, the growth factors and chemoattractant molecules incorporated into the $1.5 \mathrm{~mm}$ biodegradable PHBV/PCL vascular grafts preserve their biological activity in the grafts after their implantation into the vascular bed of laboratory animals. Promising effects of the GFmix impact on the cellular response during remodeling of the vascular tissue in situ was confirmed by the high primary patency of the PHBV/PCL/GFmix grafts within the 12-months implantation period as well as complete formation of the newly formed vascular tissues in the absence of any signs of granulomatous inflammation and a certain decrease in the rate and severity of calcification.

\section{Conflict of interest}

L.V. Antonova declares that there are no conflicts of interest related to this article. V.V. Sevostyanova declares that there are no conflicts of interest related to this article. A.V. Mironov declares that there are no conflicts of interest related to this article. E.O. Krivkina declares that there are no conflicts of interest related to this article. E.A. Velikanova declares that there are no conflicts of interest related to this article. V.G. Matveeva declares that there are no conflicts of interest related to this article. T.V. Glushkova declares that there are no conflicts of interest related to this article. Ya.L. Elgudin declares that there are no conflicts of interest related to this article. L.S. Barbarash declares that there are no conflicts of interest related to this article.

\section{Funding}

The study was supported under the fundamental research program of the Siberian Branch of the RAS, Research Projects № 0546-2015-0011 «Pathogenetic rationale for developing novel cardiovascular implants made from biocompatible materials using patientcentered approach with computational modeling, tissue engineering and genomic predictors».

\section{Information about authors}

Antonova Larisa V., PhD, the Head of the Laboratory of Cell Technologies, Federal State Budgetary Institution «Research Institute for Complex Issues of Cardiovascular Diseases», Kemerovo, Russian Federation.

Sevostyanova Viktoria V., $\mathrm{PhD}$, researcher at the Laboratory of Cell Technologies, Federal State Budgetary Institution «Research Institute for Complex Issues of Cardiovascular Diseases», Kemerovo, Russian Federation.

Mironov Andrey V., research assistant at the Laboratory of Cell Technologies, Federal State Budgetary Institution «Research Institute for Complex Issues of Cardiovascular Diseases», Kemerovo, Russian Federation.

Krivkina Evgenia O., research assistant at the Laboratory of Cell Technologies, Federal State Budgetary Institution «Research Institute for Complex Issues of Cardiovascular Diseases», Kemerovo, Russian Federation.

Velikanova Elena A., PhD, researcher at the Laboratory of Cell Technologies, Federal State Budgetary Institution «Research Institute for Complex Issues of Cardiovascular Diseases», Kemerovo, Russian Federation.

Matveeva Vera G., PhD, senior researcher at the Laboratory of Cell Technologies, Federal State Budgetary Institution «Research Institute for Complex Issues of Cardiovascular Diseases», Kemerovo, Russian Federation.

Glushkova Tatyana V., PhD, researcher at the Laboratory of Novel Biomaterials, Federal State Budgetary Institution «Research Institute for Complex Issues of Cardiovascular Diseases», Kemerovo, Russian Federation.

Elgudin Yakov L., MD, PhD, Assistant Professor, Surgery, Case Western Reserve University School of Medicine, Cleveland, Ohio, United States of America; Chief, Division of Cardiothoracic Surgery Louis Stokes Cleveland VA Medical Center Cleveland, Ohio, United States of America.

Barbarash Leonid S., PhD, Professor, Academician of the RAS, chief researcher, Federal State Budgetary Institution «Research Institute for Complex Issues of Cardiovascular Diseases», Kemerovo, Russian Federation.

\section{Authors contribution}

ALV - analysis of the results, manuscript writing.

SVV - fabrication of vascular grafts using electrospinning.

VEA - immunofluorescent analysis of the explanted grafts.

MVG - immunofluorescent analysis of the explanted grafts.

GTV - scanning electron microscopy.
MAV - grafts implantation.

KEO - histological assessment of the explanted grafts.

EYaL - analysis of the results.

BLS - interpretation of the findings and manuscript writing.

\section{REFERENCES}

1. Tara S., Rocco K.A., Hibino N., Sugiura T., Kurobe H., Breuer C.K. et al. Vessel bioengineering. Circ J. 2014; 78(1): 12-9.

2. Antonova L.V., Seifalian A.M., Kutikhin A.G., Sevostyanova V.V., Matveeva V.G., Velikanova E.A. et al. Conjugation with RGD peptides and incorporation of vascular endothelial growth factor are equally efficient for biofunctionalization of tissue-engineered vascular grafts./ International Journal of Molecular Sciences 2016; 17(11): 1920. doi:10.3390/ijms17111920. 
3. Sevostyanova V.V., Matveeva V.G., Antonova L.V., Velikanova E.A., Shabaev A.R., Senokosova E.A. et al. Constructing a Blood Vessel on the Porous Scaffold Modified with Vascular Endothelial Growth Factor and Basic Fibroblast Growth Factor. AIP Conference Proceedings. 2016; 1783(1): 020204. doi: 10.1063/1.4966498.

4. Antonova L.V., Krivkina E.O., Sevostyanova V.V., Velikanova E.A., Matveeva V.G., Mironov A.V. et al. Efficiency of using bioactive molecules in creation of functional biodegradated vascular grafts of small diameter. Siberian Medical Review. 2017;(6): 85-93. DOI: 10.20333/25001362017-6-85-9 (in Russian).

5. Glushkova T.V., Sevostyanova V.V., Antonova L.V., Klyshnikov K.Y., Ovcharenko E.A., Sergeeva E.A. et al. Biomechanical remodeling of biodegradable small-diameter vascular grafts in situ. Russian Journal of Transplantology and Artificial Organs. 2016; 18(2):99-109. (in Russian).

6. Nasonova M.V., Shishkova D.K., Antonova L.V., Sevostyanova V.V., Kudryavtseva Y.A., Barbarash O.L. et al. Subcutaneous Implantation of Poly(3-hydroxybutyrate-co-3hydroxyvalerate) and Poly( $\varepsilon$-caprolactone) Scaffolds Modified with Growth Factors. Sovremennye tehnologii v medicine. 2017; 9(2): C 7-18. (in Russian)

7. Matveeva V.G., Antonova L.V., Sevost'janova V.V., Velikanova E.A., Krivkina E.O., Glushkova T.V. et al. Modification by RGD-peptides of vascular grafts of small diameter from polyparolactone: experimental study results. Kompleksnye problemy serdechno-sosudistyh zabolevanij. 2017. (3): 13-24 (in Russian).

8. Ren X., Feng Y., Guo J., Wang H., Li Q., Yang J. et al. Surface modification and endothelialization of biomaterials as potential scaffolds for vascular tissue engineering applications. Chem Soc Rev. 2015; 44(15): 5680-742.

9. Kurobe H., Maxfield M.W., Tara S., Rocco K.A., Bagi P.S., Yi T. et al. Development of small diameter nanofiber tissue engineered arterial grafts. PLoS One. 2015; 10(4): e0120328.

10. Chong D.S., Lindsey B., Dalby M.J., Gadegaard N., Seifalian A.M., Hamilton G. Luminal surface engineering, 'micro and nanopatterning': potential for self endothelialising vascular grafts? Eur J Vasc Endovasc Surg. 2014; 47(5): 56676.

11. d'Arcy J.L., Prendergast B.D., Chambers J.B., Ray S.G., Bridgewater B. et al. Valvular heart disease: the next cardiac epidemic. Heart 2011; 97(2): 91-93. doi: 10.1136/ hrt.2010.205096.

12. Farzaneh-Far A., Proudfoot D., Shanahan C. Weissberg P.L. Vascular and valvar calcification: recent advances. Heart 2001; 85: 13-17. doi: 10.1136/heart.85.1.13.

13. Schoen F.J., Levy R.J. Calcification of Tissue Heart Valve Substitutes: Progress Toward Understanding and Prevention. Ann Thorac Surg 2005; 79: 1072-80. doi: 10.1016/j. athoracsur.2004.06.033

14. New S.E., Aikawa E. Role of extracellular vesicles in de novo mineralization: an additional novel mechanism of cardiovascularcalcification. ArteriosclerThromb Vasc Biol2013; 33(8): 1753-8. doi.org/10.1161/CIRCRESAHA.110.234146.

15. Goettsch C., Hutcheson J.D., Aikawa E. MicroRNA in cardiovascularcalcification: focus on targets and extracellular vesicle deliverymechanisms. Circ Res 2013; 112(7): 1073-84.

16. Leopold J.A. Vascular calcification: mechanisms of vascular smooth musclecell calcification. Trends Cardiovasc Med. 2015; 25(4): 267-74. doi: 10.1016/j.tcm.2014.10.021.

17. Bujan J., Bellh J.M., Sabater C., Jurado F., GarciaHonduvilla N., Dominguez B. et al. Modifications induced by atherogenic diet in the capacity of the arterial wall in rats to respond to surgical insult. Atherosclerosis 1996; 122(2): 14152.

18. Bostrom K.I., Rajamannan N.M., Towler D.A. The regulation of valvular andvascular sclerosis by osteogenic morphogens. Circ Res 2011; 109(5): 564-577. doi.org/10.1161/ CIRCRESAHA.110.234278.

19. Tintut Y., Patel J., Parhami F., Demer L.L. Tumor necrosis factor-alpha promotes in vitro calcification ofvascular cells via the cAMP pathway. Circulation 2000; 102(21): 263642. doi.org/10.1161/01.CIR.102.21.2636.

20. Cote N., Mahmut A., Bosse Y., Couture C., Pagé S., Trahan S. et al. Inflammation is associated with the remodeling of calcificaortic valve disease. Inflammation 2013; 36(3): 573 581. doi.org/10.1007/s10753-012-9579-6.

21. Fadini G.P., Rattazzi M., Matsumoto T., Asahara T., Khosla S. Emerging role of circulating calcifying cells in thebone-vascular axis. Circulation 2012; 125(22): 2772-81.doi. org/10.1161/CIRCULATIONAHA.112.090860.

22. Gossl M., Khosla S., Zhang X., Higano N., Jordan K.L., Loeffler D. et al. A. Role of circulating osteogenic progenitor cells in calcific aorticstenosis. J. Am Coll Cardiol 2012; 60(19): 1945-1953. doi.org/10.1016/j.jacc.2012.07.042.

23. Cottignoli V., Cavarretta E., Salvador L., Valfré C., Maras A. Morphological and Chemical Study of Pathological Deposits in Human Aortic and Mitral Valve Stenosis: A Biomineralogical Contribution. Patholog Res Int. 2015; 2015: 342984. doi: $10.1155 / 2015 / 342984$.

24. Pettenazzo E., Deiwick M., Thiene G., Molin G., Glasmacher B., Martignago F. et al. Dynamic in vitro calcification of bioprosthetic porcine valves evidence of apatite crystallization. The Journal of Thoracic and Cardiovascular Surgery 2001; 121(3): 500-509. doi: 10.1067/mtc.2001.112464.

25. Kudrjavceva Ju.A., Nasonova M.V., Akent'eva T.N., Burago A.Ju., Zhuravleva I.Ju. The role of suture material in the calcification of cardiovascular bioprosthesis. Kompleksnye problemy serdechno-sosudistyh zabolevanij. 2013; 4: 22-27 (in Russian).

To cite: L.V. Antonova, V.V. Sevostyanova, A.V. Mironov, E.O. Krivkina, E.A. Velikanova, V.G. Matveeva, T.V. Glushkova, Ya.L. Elgudin, L.S. Barbarash. In situ vascular tissue remodeling using biodegradable tubular scaffolds with incorporated growth factors and chemoattractant molecules. Complex Issues of Cardiovascular Diseases. 2018; 7 (2): 25-36. DOI: 10.17802/2306-1278-2018-7-2-25-36 